Natasha Randall*, Casey C. Bennett, Selma Šabanović, Shinichi Nagata, Lori Eldridge, Sawyer Collins, and Jennifer A. Piatt

\title{
More than just friends: in-home use and design recommendations for sensing socially assistive robots (SARs) by older adults with depression
}

https://doi.org/10.1515/pjbr-2019-0020

Received February 28, 2018; accepted May 15, 2019

\section{Introduction}

Deployment of physically and socially assistive robots in healthcare is increasing. Previous work has focused on designing such robots to improve the health, functioning, and quality-of-life (QOL) of people with chronic and temporary health issues and to support healthcare personnel in providing care [1-3]. While the context of use for these robots has often been limited to institutionalized care settings (e.g., nursing homes, hospitals), spurring many evaluation studies in these environments as well as the lab, researchers have recently begun to explore the use of assistive robots in single family homes to provide daily therapeutic support and preventative assistance to patients and caregivers. Initial implementations of assistive robots in homes show promising health benefits, such as addressing loneliness and social isolation using companion robots [4-7], providing medication and event reminders [5, 8], and monitoring and predicting users' health status with sensors accompanying the robot $[4,5,8]$.

Increasing interest in developing robots for use in homes as well as institutions parallels a broader trend in healthcare towards patient- and community-centered care [9]. As healthcare shifts away from the traditional medical model centered on diagnosis and treatment of disease, community-based interventions are becoming more common, with research showing they improve clinical outcomes and reduce health care costs associated with long term institutionalization [10-12]. This shift underscores

\footnotetext{
Selma Šabanović: School of Informatics, Computing, and Engineering Indiana University, USA

Shinichi Nagata: School of Health Science and Wellness Northwest Missouri State University, USA

Lori Eldridge, Jennifer A. Piatt: School of Public Heath Indiana University, USA

Sawyer Collins: School of Informatics, Computing, and Engineering Indiana University, USA
}

\footnotetext{
*Corresponding Author: Natasha Randall: Indiana University Bloomington, USA; E-mail: nprandal@iu.edu

Casey C. Bennett: College of Computing and Digital Media DePaul University, USA
} 
the potential for socially assistive robots (SARs) as a health intervention tool within the current healthcare landscape.

The use of robots in home care brings up new questions for the fields of SARs and human-robot interaction (HRI), including whether older adults will accept SARs in their homes, whether and how they will use SARs without clinical supervision, and whether SARs that were beneficial in institutions will have similar effects in homes. In this paper, we seek to understand how SARs might be used in homes to support the care and therapy of older adults with depression. We focus on older adults with depression because of its high incidence rate among older adults [13], and its correlation with higher rates of institutionalization [14]. Preliminary data suggests that SARs may have therapeutic benefits in this area $[2,3]$, including reducing loneliness [15], improving mood [16], increasing socialization [17], and relieving caregiver burden [18]. There are, however, still many open questions regarding the design characteristics and ways of incorporating SARs into home care that would be most useful for assisting older adults with depression.

To address these open questions, we conducted focus groups with older adults diagnosed with major depressive disorder (MDD) and with clinicians and care managers who work with this population, as well as a month-long in-home intervention with these same older adults, to understand their evaluations of existing SAR technologies in use and gather design ideas for future SARs they could use in daily care and therapeutic practice. We particularly focused on the subcategory of companion SARs, as those were most relevant to the concerns older adults with depression had mentioned in previous participatory design studies $[19,20]$. Along with appropriate functions of the robot and the robot's fit in people's homes, we also wanted to know how sensors accompanying the robot might be used to support personal and clinical care for older adults with depression. Previous work showed that one of the challenges for potential users participating in robot design activities is their lack of experience with these emerging technologies [21]. To address this issue, we provided older adults with depression with the zoomorphic SAR Paro (Figure 1) - a robotic seal often used in eldercare - to use in their homes for one month between initial and final focus groups as a way of providing them with hands-on and contextually situated experience with SARs that could inform their evaluations. Having Paro in the homes of potential users allowed us to learn about emergent practices and interactions people had with companion-type robots. It also gave participants enough long-term experience with the robot to provide additional insights in the final focus group on what they found desirable and what they wanted

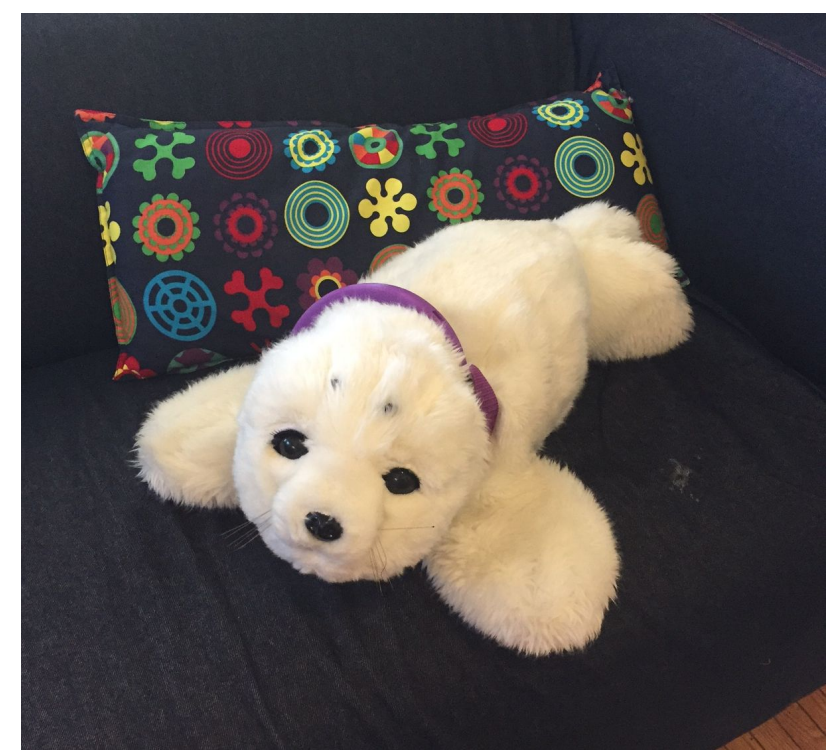

Figure 1: Paro: a socially assistive, seal-like robot.

to amend. Additionally, we gauged people's perceptions of having data about their interactions collected via sensors onboard the robot and made available to them and others (e.g., therapists, family) as an additional intervention mechanism and explored the potential use of such sensors in the course of depression care and therapy.

The first contribution of our work is in executing a long-term, real-world study that uses a healthcare robot in the home environment, suggesting that use of such agents for preventative in-home healthcare may be possible. Second, there have been no studies of in-home use with individuals who have MDD (depression) as their primary diagnosis, and our paper illustrates the ways in which the design and use of SARs for this population should be situated in their daily concerns. Third, while there has been a lot written on the use of sensors for healthcare, our work provides insights into how data from sensors onboard SARs correspond to self-reported use, and how sensors could enable SAR behavior adaptation in a clinically meaningful manner [7]. Our study shows the value of extended experience with emerging technologies like SARs for informing user contributions to the design of robots, as some design preferences and suggestions changed after in-home use, and both researchers and participants had a clearer idea of how requested robot functionality might be implemented after the study. Lastly, we believe a novel empirical contribution is in determining that, while companionship may be sufficient to inspire continued intention to use SARs for those with mental health issues in institutions, it is not always sufficient to inspire intentions for long-term home use in this population. 
Our results are organized as follows: Section 4.1 and 4.2 discuss attitudes toward the robot and SAR design ideas presented by older adults and staff pre-intervention (4.1) and post-intervention (4.2). Section 4.3 connects design ideas to the lived experiences of participants during the intervention. Section 4.4 presents our robot perception survey results and connection with pet ownership, and section 4.5 presents robot use based on sensor data and correlations with intention to use and patterns of interaction.

\section{Background literature}

\subsection{Depression in older adults}

Depression has a high incidence rate (15-20\%) among older adults [13], and commonly precipitates deterioration in individuals' physical and mental health. People with depression are three times less likely to adhere to treatment regimens, and more likely to be readmitted to the hospital than other patients [22]. Per-person cost of healthcare for older adults with depression is accordingly 47-200\% higher than their counterparts without depression [23]. 47.5\% of older adults with depression are also diagnosed with anxiety [24]. A major component of depression in older adults is the lack of social interaction, combined with chronic loneliness [25]. Pet ownership has been associated with decreased depression among women and single individuals [26]. Treatment for depression is commonly performed through clinical visits and medication, but can also include supplemental treatments such as animal-assisted therapy, which has been found to decrease symptoms of depression in institutions [27].

\subsection{Use of SARs with individuals with depression}

There is limited research examining the effects of SARs on depression. Our mental health survey results from this research project, reported in Bennett et al. [4], showed a reduction in depression in older adults, but failed to find an improvement in loneliness or functioning. Where other research is available, it has looked at therapeutic effects on depression in individuals with dementia. Wada [17] found improved overall mood and lowered depression scores, as measured by the Geriatric Depression Scale (GDS), after a one-year deployment of Paro at a care facility. In a 12-week study at a retirement home, Robinson, et al. [15] found no significant differences in GDS scores between intervention and control groups after adjusting for baseline scores, but did find that individuals' loneliness was significantly reduced in the intervention group when compared to control. There is some evidence that SARs do decrease loneliness and improve social connections, mood, and health among older adults $[2,28]$. Paro has been one of the main robots used in studies showing these positive effects.

Prior work on designing robots for older adults with mental health issues suggests that it may be appropriate to use robot companions in lieu of pet therapy $[29,30]$. Pet therapy has had success as a treatment for older adults with depression [31,32], but pets require a high level of care that mental and physical issues sometimes prevent. In prior participatory design studies with older adults with depression, several participants lost access to their pets during the study due to deteriorating health [15]. Money needed to care for pets may also make it difficult, if not impossible, for low income individuals to own animals. Additionally, not all residences allow pets, and there may be allergy concerns.

Though counselling services are often available for individuals with depression, meetings with clinical staff typically occur every one or more weeks, leaving ample opportunities for supplemental intervention. Also, though medication is efficacious for many adults, there is often additional benefit from receiving concurrent treatments [33, 34]. This makes the use of robots and accompanying sensors to monitor and provide appropriate personalized interactions for older adults potentially advantageous.

\subsection{SARs in the homes of older adults}

Several studies on the therapeutic use of SARs have evaluated their effects on the mental and physical health of older adults in institutions $[35,36]$, but few have focused on SARs in single family homes. This is an important distinction, however, as people have more freedom to interact as they like with personal robots in the home than they would in institutional contexts. They may also form tighter bonds with robots in their home, seeing the robot as their companion rather than a shared artifact.

One of the few studies of long-term in-home SAR use by older adults was by Liang et al. [37]. They conducted a six-week in-home intervention with Paro, coupled with institutional use, for dementia patients. Participants who had less severe dementia symptoms benefitted the most from Paro's use in this case. Additional studies have probed how other robots might support the health of older adults in the home. Coradeschi et al. [38] used GiraffPlus, a telepresence robot, to facilitate in-home communications between patients and care providers. As a supporting feature, they allowed friends and family members 
to interact with older adults through the GiraffPlus system. Another robot, Care-O-Bot II, was used to provide physical assistance, as it supported walking and item handling by older adults [39]. Therefore, both socially and physically assistive robots have found their way into the home.

With older adults' desire to "age in place", more studies are necessary to determine how to accomplish this goal, including using new technologies. The present study is the first to use SARs in the homes of persons with clinical depression as a primary diagnosis, with special emphasis on how robots should be designed to maximize utility for this population.

\subsection{Sensors in in-home healthcare}

There is extensive literature on the use of environmental and wearable sensors to track the health status and behavior of older adults [40-42]. In the case of older adults with depression, for example, motion density maps based on passive infrared sensors placed in users' homes have been shown to correlate with health assessment scores [43]. Sensors that can alert caregivers to distress (such as a sudden blood pressure drop or changes in behavioral patterns via activity monitoring) have also been placed in the homes of independently living older adults [44]. Activity monitoring in this case was done via infrared sensors around the home, allowing older adults to maintain some independence and privacy, with caregivers getting alerts only when necessary. There is less knowledge on how sensors onboard SARs can be used to collect clinically useful data, or how environmental and wearable sensors can enable SAR behavior adaptation in a clinically meaningful manner $[5,8,45]$.

The use of social robots in the home brings about unique concerns over user privacy and questions about data sharing, as robots may have many sensors that are required for their functioning and collect information about people's behaviors in an intimate space. People who have developed privacy-enhancing behaviors in the presence of more overt sensors may be less aware of or concerned about possible privacy infractions by technologies designed as social entities, whose social presence may obscure the identity of the people receiving the data [46]. On the other hand, this fact might make sensors onboard SARs particularly useful, as patients might not attempt to actively obscure their behavior.

\section{Methods}

\subsection{Participants}

Participant recruitment took place in a midwestern city in collaboration with a local outpatient mental health clinic. The recruitment criteria for older adults specified participants should be over 55 , live independently in their own homes, have an active depressive disorder diagnosis, and present with a co-occurring physical illness. We recruited participants who were over 55 , instead of 65 , as people with depression show cognitive signs of aging earlier than those without depression [47]. Those who had also been diagnosed with psychosis (e.g., schizophrenia) or dementia were not eligible for the study.

In total, 10 older adults were recruited. They ranged from 56 to 67 years old, with an average age of 62.8 years. While recruitment of both male and female participants was attempted, no males volunteered for this study; therefore, all participants were female. This might be due to the larger overall incidence of depression in the female population $[48,49]$, or to females' greater willingness to interact with companion robots [50].

The lifestyles of older adult participants were diverse (see Table 1). Several lived alone, while others lived with significant others, roommates, or children. Some had pets. Their access to social support also varied widely. They had different secondary diagnoses, including anxiety and physical ailments. All older adults were low-income, perhaps due to the location or method of recruitment, and all but one (P10) were unemployed.

Additionally, 4 male and 4 female clinicians were recruited from the same local outpatient mental health facility. They worked in diverse healthcare roles, including care coordinator, therapist, and crisis worker. All had worked with our target population of older adults over 55 years of age, who were living independently and had been diagnosed with MDD.

\subsection{Study design}

The study consisted of initial focus groups with older adults and staff, a SAR in-home deployment, and concluding focus groups. 
Table 1: Older adult participant demographics.

\begin{tabular}{|lllll|}
\hline Participant & Age & Cohabitants & Pets & Social support \\
\hline P1 & 65 & Grandson & Dog & $\begin{array}{l}\text { Daughters live nearby and stop by frequently. Grandkids in home. Both positive and } \\
\text { negative (stressful) interactions with family. }\end{array}$ \\
\hline P2 & 62 & None & Dog & Daughter lives in town, and they frequently get together. \\
P3 & 67 & $\begin{array}{l}\text { Husband, } \\
\text { Son }\end{array}$ & Dog & $\begin{array}{l}\text { Loving relationship with husband and son. Speaks regularly with other son who lives out of } \\
\text { state and his children visit once a year. Attends church services weekly. }\end{array}$ \\
\hline P4 & 67 & None & Dog & Has a great relationship with daughter who lives nearby. They frequently interact. \\
\hline P5 & 62 & None & None & $\begin{array}{l}\text { Sister calls to chat sometimes but the two do not live near one another, so they don't see } \\
\text { each other. Her neighbor checks on her periodically. }\end{array}$ \\
\hline P6 & 67 & None & None & Very little support. Not close to family and had no close friends. \\
\hline P7 & 60 & None & None & Her father and sister live in the area, and they all frequently get together. \\
\hline P8 & 61 & None & None & Almost no social support. No one, besides her aids, who visits or calls. \\
\hline P9 & 56 & Grandson & Cat & Has a boyfriend. Daughter lives in the area, though their relationship is often fraught. \\
\hline P10 & 61 & Roommate & Dog & Has a roommate and is supported by family and friends. \\
\hline
\end{tabular}

\subsubsection{Older adults}

\section{Pre-intervention focus groups}

The two initial focus groups were conducted with three to six older adults participating in each. The purpose of the first focus group was to introduce participants to several SARs ( $\mathrm{Mabu}^{1}, \mathrm{Jibo}^{2}, \mathrm{Paro}^{3}$ and Buddy ${ }^{4}$ ) through videos and to get their initial feedback on such robots through group discussion. Participants discussed various features of the SARs and whether and how they thought SARs could be useful in their lives. Additionally, they were able to interact with Paro in person during the workshop. Participants were asked to comment on how they thought Paro worked (e.g., which sensors it had and how it responded) and were encouraged to share their thoughts on what it might be like to have Paro in their homes.

The second focus group was conducted to gauge participants' reactions to sensors that would be used with Paro during the study. These included sensors embedded in a 3D printed collar worn by Paro and a Jawbone activity tracker worn by participants. The sensor collar was designed to collect data on ambient light, ambient sound, and interaction with the robot, while the Jawbone collected sleep and activity data. We used a sensor collar as opposed to embedded sensors within the robot, as sensor data collected directly by Paro is proprietary. Participants were given the sensors to examine and were shown visualizations of the types of data the sensors could collect. Researchers asked participants how comfortable they were having these in their home. They also inquired as to whether the information that would be collected by the sensors would be useful

\footnotetext{
1 https://vimeo.com/138783051

2 https://www.youtube.com/watch?v=3N1Q8oFpX1Y

3 https://www.youtube.com/watch?v=V-WTCm7kOP0

$4 \mathrm{https} / / /$ www.youtube.com/watch?v=51yGC3iytbY
}

to them, and what they thought about giving others access to this information (e.g., therapists, family members).

\section{In-home intervention}

After completion of both focus groups, participants were given the Paro robot to use in their homes for four consecutive weeks. We chose Paro as the companion SAR because there is previous evidence that it can help alleviate loneliness and improve mood among older adults [15], it is FDA approved as a biomedical feedback device, and it is a commercial device that is easy to use and technically robust, making breakdowns less likely. Older adults with depression in previous participatory design studies also showed a clear preference for using Paro in their homes [20]. Participants were instructed on how to turn the robot on and off and use it safely, but were free to choose how and when to interact, except they were asked not to sleep with it (for safety reasons) and to not take it out of the home (to help prevent loss and damage). At the commencement and conclusion of the study, researchers administered self-report surveys to gauge participants' mental health and wellbeing (i.e., PHQ-95, WHOQOL-BREF (WHO, 2004) ${ }^{6}$, UCLA loneliness scale $^{7}$, OQ-45) as well as two robot perception surveys (i.e., Almere, Godspeed) [51, 52]. We used a slightly modified version of the Almere by removing four questions (e.g., pertaining to the robot being human-like) that did not make sense in the context of our study. Three of these questionnaires were also administered weekly (PHQ-9,

\footnotetext{
5 http://www.phqscreeners.com/sites/g/files/g10016261/f/201412/ PHQ-9_English.pdf

6 http://www.who.int/substance_abuse/research_tools/en/ english_whoqol.pdf

7 http://fetzer.org/sites/default/files/images/stories/pdf/ selfmeasures/Self_Measures_for_Loneliness_and_Interpersonal_ Problems_UCLA_LONELINESS.pdf
} 
WHO, UCLA loneliness scale). These provided data on participants' changing mental and physical health status, their interactions with the robot, and their experience using SARs. Our aggregate mental health questionnaire results presented in Bennett et al showed that Paro may alleviate symptoms of depression as measured by the PHQ-9 when used as a supplement to existing treatment [4]. Weekly semi-structured in-home interviews were also conducted to learn more about participants' activities. A daily five-minute phone call with participants was attempted to note changes in their mental and physical health, and interactions with Paro (though participants did not always take the call). Data from sensors in Paro's collar (ambient light and sound, accelerometer) tracked participants' interactions with and around the robot, while Jawbone activity trackers collected data on their sleep and physical activity levels for the duration of the in-home study. Sensor data results, also reported in Bennett et al [4], showed that changes in depression status could be predicted with $\sim 74 \%$ accuracy. Participants continued their previously prescribed treatments during the intervention.

\section{Post-intervention focus group with older adults}

Following Paro's removal from their homes, participants were invited to a final focus group to discuss their experience, thoughts on SARs usefulness in their life, Paro's form and features, further design ideas that might extend beyond Paro's capabilities, how the sensor data might be useful to them, and any privacy concerns related to having sensors in the home or sharing the collected data. Focus groups were conducted with two to three participants in each. Seven of the ten participants who completed the inhome study attended a session. Of the three participants who did not attend, one had scheduling conflicts, and the other two were unresponsive to our repeated attempts to reach them. Based on our experience recruiting for this project, speaking with clinicians, and working with depressed older adults more generally, it is not uncommon for members of this population to experience fluctuations in depression and become unreachable when their depression worsens.

\subsubsection{Clinical care staff}

Pre-intervention focus groups with staff

The focus groups were conducted in two sessions, one with 6 staff members and the other with 2. Participants were introduced to Paro through video[? ] and in person. They were also shown various sensor cards, depicting sensors that could be used in robots and briefly describing their function [53]. Researchers asked staff if they foresaw any benefits or issues with its use by older adults with depression, questioned them as to whether and how they would incorporate Paro into their practice, and facilitated discussions about sensors and their potential use in treatment.

\section{Post-intervention focus groups with staff}

After the final focus group with older adults, a concluding focus group was conducted with five out of the original eight staff members. This served as a debriefing of what happened when older adults had Paro in the home. Therefore, anonymized sensor data from the collar and activity tracker were shared as well as ways in which participants engaged with Paro. Staff commented on how they might want to see and use this information to support patient care, both as information to discuss during therapy sessions and by using the sensor data to have SARs adapt their behavior to the patient's conditions and needs. Information on additional sensor data staff wanted to have was also collected, in addition to capabilities they would want to see in a SAR for older adults with depression.

\subsection{Data analysis}

\subsubsection{Focus group data}

An inductive approach to thematic analysis was performed in order to code the data and identify emergent themes. For focus groups with older adults, a total of 443 codes were created after line-by-line coding of the data. These included codes pertaining to what participants liked and wanted to change about Paro, what they wanted in a social robot more generally and their feelings post-study, whether they wanted access to sensor data and what they would like to use it for, and their feelings on sensor data sharing. $15 \%$ of this data was randomly selected and coded by a second researcher. The pooled kappa was 0.70 . For staff, a total of 646 codes were applied after line-by-line coding. They were then categorized into 92 different code types and divided into 9 themes. The three most common themes were: a) using sensors to enable the robot to respond based on older adults' health, b) using sensor data to monitor mental health and inform therapy, and c) using sensors for more general health monitoring, such as sleep and smoke detection. 


\subsubsection{In-home interview data}

Deductive and inductive approaches to coding were coupled to thematically analyze the data collected through telephone calls and interviews in participant homes. This allowed coding of concepts of pre-established importance as well as recurring themes that naturally arose from the data. In the end, 22 codes were created. This included codes for physical affection toward Paro, anthropomorphization, positive and negative emotional changes (due to Paro), and positive and negative interactions with other people. To check interrater reliability, $20 \%$ of the data was randomly selected and coded by a second researcher. Discrepancies were discussed. Cohen's kappa ranged from 0.74-1.0 for the 22 codes.

\subsubsection{Sensor data}

Sensor data was modeled to test its utility for predicting participant attitude changes over time, as measured by the Almere scale. This differs from previous work, where we focused on using sensor data to predict changes in clinical scores [7]. Sensor data (described in section 3.2.1.2) from the robot collar and the wearable Jawbone were utilized, along with baseline outcome measures (to control for differences in participant functioning and symptom levels prior to intervention).

The first step of this modelling entailed a sensor fusion process. Data was first thresholded using a low-pass filter to remove noise from the signal. For example, this excluded small vibrations from the accelerometer (motion) data. Positive detection of various signal modalities (motion, light, sound) was then calculated using the average across a 30-frame moving window (i.e., approximately 3seconds) to minimize false positives $[54,55]$. Next, interaction activity was identified by the presence of multiple positive sensor modalities at any given timepoint. Finally, the fused sensor data was aggregated temporally (hourly, daily, weekly) to look for circadian rhythm patterns of human-robot interaction.

After the sensor fusion step, data was modeled in two ways. First, we ran a basic correlational analysis between all the variables. Second, the data was run through a machine learning analysis via the Python Scikit package (version 0.20.2, https://scikit-learn.org) and WEKA [56], attempting to predict the change in the Almere attitude scores pre vs. post intervention. Modeling methods included Random Forests, Neural Networks, Gradient Boosting, and Support Vector Machines [57], which were evaluated using 3-fold cross validation (given the limited sam- ple size). Feature selection was also conducted to identify features most highly related to attitude changes, using the Relief-F algorithm [58].

\section{Results}

\subsection{SARs and sensors - attitude and design ideas prior to home use}

\subsubsection{Older adults}

\section{SARs}

Participants' initial perceptions of Paro were captured during the first focus group, which was conducted prior to participants' in-home use of the robot. Six out of ten participants [P1, P2, P6, P8, P9, P10] immediately took to Paro and exhibited excitement to have it in their homes, as shown in the following quotes.

"I have a little stuffed bear that I keep on my bed. And he doesn't respond to me, so I was thinking that would be kind of nice to have that!... I was thinking, if I could have one of those I think I would probably like to have one!” (P8)

"I'd carry that thing everywhere... Oh, I think it's awesome. I love it.... I'm excited. I am, I'm excited... I wouldn't change a thing. Because it's already doing most of what some people don't even have. And you get something like that and it's new and that's a friend forever." (P1)

"I'm looking forward to having one to take home with me too. We're just really excited about getting our little seal.” (P6)

The remaining four participants either expressed negative [P3, P4] or neutral [P5, P7] sentiment toward using Paro. Specifically, P3 and P4 did not see any personal value to using Paro, stating that it was not for them.

"I still have dogs so I don't know, it'd be quite a jump for me to go from a dog to a robot." (P3)

"Would I want one? Not particularly. Maybe later, at some point. But right now, no." (P4)

Though only six participants saw Paro as potentially benefitting them, almost all participants (8/10) wanted a home robot of some sort. All eight mentioned they would want it to offer reminders - for example, for appointments, taking medication, and to eat and exercise. Using the robot to contact the hospital or a designated contact in medical emergencies was requested second most often $(\mathrm{N}=6)$, and participants wanted the robot to learn and change its behavior to suit their needs and the context $(\mathrm{N}=4) .4$ out of the 10 participants also spoke about their need for the 
robot to offer companionship. An ability to communicate (verbally or nonverbally) $(\mathrm{N}=4)$, be versatile and customizable $(\mathrm{N}=6)$, and function as a security system $(\mathrm{N}=5)$ were also in high demand.

Participants did have concerns about using, or their ability to use, home robots. Their prime concerns included the robot being too technically challenging for them to use $(\mathrm{N}=3)$, high cost $(\mathrm{N}=4)$, privacy and hackability of the technology $(\mathrm{N}=6)$, and competing desired usage between users $(\mathrm{N}=4)$. Concerns about Paro specifically were similar to those about home robots in general (though they felt confident in their ability to use it). Though their discussions about Paro were mostly positive, they mentioned wanting to change its form to something more familiar (cat, dog, or stuffed animal) $(\mathrm{N}=2)$ and one person expressed worries about it being white, as it might get dirty.

\section{Sensors and sensor data}

Most of the older adults were willing to share sensor data with medical staff and family $(\mathrm{N}=7)$. Fewer agreed with sharing data with a third party robot developer $(\mathrm{N}=2)$. They wanted to be able to control who saw their data, but saw data sharing overall as a positive feature. They also wanted access to their own data, mainly to track their sleep, changes in their motivation, and calorie consumption.

\subsubsection{Clinical care staff}

SARs

Staff were positive about the potential for using Paro to assist in treatment and care of certain populations, mainly older adults and those with dementia, both in and out of nursing homes. They particularly saw it as beneficial for those with depression and other emotional issues, such as anxiety and PTSD ( $\mathrm{N}=3)$.

“[A lot of our patients] live where they can't own a pet [and they] would really love to own [one], so I'm like you could get a fish, but they want a dog or a cat. They just can't where they're at, and so I think that they would probably enjoy it." (Staff 2)

"Just the sensory deprivation that comes with isolation, being able to interact with something that kind of resembles another living creature." (Staff 1)

Staff's suggestions for home robot features for older adults with depression focused on four areas: increasing interaction and socialization, user recognition and personalization, sensor customization, and communication to an appropriate contact (hospital, police, designated contact) when individuals were in mental or physical danger. They wanted sensors to be able to identify the user's behavioral patterns and emotions, and to be customizable for each client. These ideas related to goals therapists had for clients, including increasing socialization, increasing hygiene and personal care during low periods, increasing exercise and movement, developing coping skills, establishing a routine, and remembering to take medications.

Though staff liked Paro overall, they saw several ways it could be improved for their patients. As previously mentioned, they wanted to be able to customize the robot's response and sensors based on patients' needs $(\mathrm{N}=7)$. Like older adults, they also wanted it to be a more familiar animal, either a cat or a dog $(\mathrm{N}=3)$. They wanted to be able to prompt older adults into interacting with the robot, and for the robot to prompt older adults into interacting with other people. They found it useful that the collar designed to accompany Paro sensed light, because individuals experiencing high levels of depression often keep their homes darker, and wondered if Paro could be solar powered, which would prompt users to go outside. Some wanted Paro to require care $(\mathrm{N}=2)$, reasoning that it would give persons with depression a sense of purpose and be helpful in spurring people to action when they were emotionally low. Furthermore, since sleep is often jeopardized in this population, they thought it valuable if the robot could help patients sleep, potentially by playing white noise or helping them with relaxation techniques, and if sensor data could report to patients and therapists how well individuals slept.

Like older adults, cost was staff's top concern surrounding long-term use of robots, such as Paro, in the home. They also expressed organizational concerns if they were to adopt them into practice, worrying about liability issues for their center and individual therapists that might arise with its use as well as damage to the robot. Despite these potential drawbacks, they expressed a number of ways they could see incorporating robot home use into the care routine for older adults with depression, such as implementing a clinician-controlled check-out $(\mathrm{N}=2)$, using it as a reward for patients making progress toward their goals $(\mathrm{N}=4)$, using its accompanying sensors to check that patients are meeting their goals $(\mathrm{N}=5)$, and using it as a tool to open a dialogue between the therapist and patient $(\mathrm{N}=4)$.

\section{Sensors and sensor data}

Staff also discussed what kind of information they would want sensors to detect. For example, three staff members mentioned detecting the environment in some way, partic- 
ularly dust, temperature, or humidity. Home control was also mentioned (i.e., managing temperature or light control). They thought it would be useful if the robot could detect the area around it and offer alerts for emergencies (fire, security) or regular use (someone at the door). These functions were thought to be useful for SARs to be used by their patient population more generally.

More commonly, staff mentioned wanting the robot to be able to identify behavioral patterns $(\mathrm{N}=7)$, along with speech patterns and emotions $(\mathrm{N}=5)$ via its sensors. They expected this to help with monitoring patients' mental health status. It was noted that these sensors should be customizable for each patient. "Our whole focus here is to treat the individual as a unique set of goals, objectives, and problems. Paro would have to represent that in order for us to effectively use it" (S4).

\subsection{SARs and sensors - attitude and design ideas after home use}

Below we present findings about participants' perceptions of the robot and sensors from interviews with participants during the in-home intervention, post-intervention participant workshops, and post-intervention workshops with staff. 7 out of 10 of the original older adults and 5 out of 8 of the original staff attended the final workshops.

\subsubsection{Older adults}

\section{SARs}

Five out of ten participants remained constant in their original attitudes toward Paro during the course of the in-home intervention, as expressed in the initial focus groups. P2, P6, P9, and P10 continued to display positive feelings about Paro that grew during the course of the study. In contrast, P4 remained uninterested, believing that Paro was not necessary for her and expressing at the final workshop, "I really didn't miss him".

However, some participants' attitudes toward the robot changed dramatically from their first encounter. P5 and P7, who did not express specific attitudes toward Paro to begin with, both ended up becoming enamored with it.

"I'm usually telling her what a good girl she is and what a pretty girl she is. And I tell her I love her." (P5)

P3 had a near immediate attitude change after Paro had been in her home for the first week. She began to display emotional attachment toward the robot, and discussed early on that she would miss it when it was gone.
She also said it comforted her in times of stress. During the final focus group, which occurred months after the intervention for this participant, she continued to exhibit attachment and iterated that she still missed Paro. Her attitude about having Paro in her home before the study, which was quite negative, transformed once Paro became a member of her household.

P1 was the opposite case, going from a positive to a predominantly negative view of having Paro in her home.

"It's like a dog.... I don't really want to keep the dog, but I don't want to take him to the pound and have him put down neither. So it's a lot of responsibility." (P1)

P8's attitude toward Paro also changed, but this change did not occur while Paro was in her home. It was only after Paro's removal that she began to think Paro wasn't that special - it was no different from her stuffed "talking” bear Koda (which says "I'm Koda", "I like climbing trees”, etc. when you press its ear) that she had had for years.

As far as the robot's design, in the final workshop many participants still wanted Paro to be in the form of a dog or cat and for it not be white, as they had expressed in the initial focus groups. Other design ideas emerged only after in-home use of the robot. For example, two participants wanted a robot with a more versatile form, so it could either be set to sit or stand, and for it to be more moldable, so it could wrap around their body more comfortably.

As opposed to participants' initial desire to enable multi-person usage in the initial focus group, there was a shift to users viewing Paro as theirs. They wanted to maintain control over whether others were allowed to interact with it. P10 stated, "when I shared him with my husband it was me sharing him." P3 echoed this sense of protectiveness and ownership over Paro. Both participants $(\mathrm{N}=5)$ and staff $(\mathrm{N}=4)$ had mentioned detecting dust, temperature, humidity, pressure, and/or home management in the initial workshops, but after implementation these things became less important for both groups. This is probably because other functionality was seen as more important overall but also seemed to be more in-line with the features of a companion type robot.

The ways in which the robot needed to be more customizable for participants also became apparent after they had experience using it in the home. For example, some loved the duration, pitch, and volume of its cries, while another found them too loud, frequent, and shrill. This was enough to eventually prevent one user from turning him on: "After a while I just couldn't listen to him anymore." (P8) Some found its behavior diverse enough to not get bored, but most wanted the robot to learn and change over 
time. This adaptation was seen as best in response to their idiosyncratic patterns. P3 even wanted the robot to sense her emotions in order to respond appropriately. Even participants who found Paro's behaviors robust enough, still conceded they would probably prefer a robot with the ability to adapt.

While Paro was found to be responsive for the most part, older adults also wanted it to have a motion sensor so it would become active automatically when they entered the room, even if it was off $(\mathrm{N}=3)$. This request was in part due to the fact that it only responded to sound and not motion, and in part due to Paro's weak battery life, which meant they were likely to turn it off went not in use. Its battery only lasted for two hours before needing to be recharged, a problem mentioned multiple times by several participants $(\mathrm{N}=4)$. They wanted it to last four hours at minimum, with a preference for longer. Having to plug it in constantly was a major impediment to its use, though many older adults still interacted with the SAR while it was charging. It is helpful that newer versions of Paro have a longer battery life of up to eight hours, which addresses this issue.

In line with the particular issues associated with this user group, the companionship that Paro offered was often cited as a favorite feature $(\mathrm{N}=4)$. When we asked participants what affected them most during the study, they answered:

"Not being alone." (P9)

"Yes - yes not being alone. Even though I have a roommate but I felt PARO was more mine, you know?" (P10)

Beyond use as a socialization partner and mediator, users wanted a home robot to be able to offer other functionality (such as contacting someone if they were unwell, use as a security system, and reminders), even if its primary purpose was companionship $(\mathrm{N}=4)$.

“I think I wasn't as fascinated with him as I was originally... I just felt like he needed to have done something else.” (P8)

"Can you imagine what [Paro] would need to do in order to keep you engaged for longer?” (Researcher)

"I wished he could have reminded me of appointments." (P8)

"So having some kind of functional use basically other than just being there.” (Researcher)

"Or some kind of alarm." (P2)

"Yeah" (P8)

They did not feel these features would interfere with its primary function as a companion. Companionship, therefore, may be a necessary but not sufficient function for a robot for long-term home use with many members of this population.

\section{Sensors and sensor data}

Only one participant reported being somewhat aware of the sensors in her home gathering data. The others either did not notice or did not care. This is likely in part because the sensors were designed to detect low level information (e.g., if sound was present and at what level, as opposed to recording what was said). The main time participants became cognizant of the sensors in the collar was when they had to swap the batteries, which they had difficulty doing, as the device could be finicky and the batteries needed to be changed every few days. Three participants also had issues putting on and taking off the Jawbone fitness tracker, and participants often forgot to wear it. Therefore, we found that wearable sensors were not a good way to incorporate sensor technology long-term for this population, and that sensors should either be onboard the robot or located in their external environment, with minimal maintenance required by users.

Participants ( $\mathrm{N}=5$ ) were willing to share their data with third party robot developers to improve their in-home experience, though one person was opposed. This presents a shift from attitudes expressed at the initial focus groups, where most were uncomfortable with this idea. Three participants actually did not care who got the data, though the others talked about placing restrictions on it. Most wanted a designated contact (e.g., friend, family member) to be able to see their data in an interpretable way. This also supports staff's desire to build a support system between older adults and other people. Four people wanted to share this information with family, and two were opposed. This made sense, as they were close to their families to varying degrees. All participants present $(\mathrm{N}=7)$ were fine with their therapists and staff receiving this information.

Five individuals also thought the data would allow them to meaningfully monitor their own habits. They were particularly interested in using sensors to obtain information on their sleeping habits $(\mathrm{N}=3)$ and to use it to motivate them to be more active $(\mathrm{N}=2)$. Additionally, they were interested in sharing it with clinicians, and using it to help manage their addictions and show they correctly followed recommended use of the robot.

\subsubsection{Clinical care staff}

\section{SARs}

In the final staff workshop, one person wanted older adults to have to do something to actively care for the 
robot, otherwise they might just be low together. Additionally, the robot actively mirroring the participant was suggested as a way to make the patient more cognizant of their own emotions, given that they were told about this functionality in advance. This might cause them to seek help during particularly low moments or do something to regulate the robot's (and their) emotions. This idea of using a companion robot as a mirroring tool seemed to be popular, with another staff member agreeing that it could even be used to prompt and discuss hygiene. For instance, they might be required to brush Paro before they brush their own hair. As well, therapists could tell how often Paro was groomed and this could lead them to segue into a discussion of participants' own grooming habits while in session. "Sometimes [poor personal hygiene and grooming] is a warning sign.” (S3

\section{Sensors and sensor data}

In addition to customizing the robot with patients, staff also wanted to be able to customize sensors for each patient. Still, there were some features they saw as being useful for all patients, such as providing sleep data $(\mathrm{N}=3)$ and identifying behavioral patterns $(\mathrm{N}=2)$. Being able to prompt older adults into interacting with the robot $(\mathrm{N}=1)$ and having the ability for others to communicate through the robot $(\mathrm{N}=1)$ were also mentioned. They saw this data, if it was presented in a visually and easily interpreted format, as able to feed into therapy in two main ways. First, they thought having the information present would allow them to open a dialogue with patients. "I think more data is good also because it gives clients more opportunity to participate in their treatment; otherwise, they feel like they have to say what they think we want to hear or they just shut down often." (S5) Second, they liked that they could observe changes in patient's activity, prompting intervention or discussion. Related to this, they wanted to set baseline patterns (e.g., sleep, appetite), so anything out of the ordinary would be easily brought to their attention. They felt that this could be used to demonstrate to the patient objectively that they were improving as well.

\subsection{Connecting design ideas to findings from the in-home intervention}

Below we discuss how functionalities requested by participants were relevant to different aspects of older adults' daily lives. Suggestions are also offered for how to implement features when designing home robots for this population.

\subsubsection{Companionship}

During the in-home intervention, older adult participants described how important companionship was to them, and in many situations, said Paro was able to meet that need when it was lacking. All participants had many moments where they felt lonely, sad, alone, or bored, and described that having Paro around alleviated the intensity of these feelings or made them dissipate altogether.

"He's always something that I can look at and feel good about myself and having him around. Cuz sometimes that helps with sadness.” (P2)

"Yes. I love the robot. It keeps me company. I feel safe with it." (P7)

Participants were likely able to view Paro as a companion because they endowed him with emotions and likened him to the people and pets around them. Eight of the ten participants named the robot something other than Paro, the name we called it in the focus groups. Having the robot in their homes, a more intimate space where it was viewed as belonging to them, seemed to foster the view of Paro as a friend or family member.

Furthermore, several participants mentioned that Paro increased the frequency with which they interacted with other people, as other people were drawn to ask about Paro or interact with it, starting a conversation. Participants nearly always enjoyed this; however, P1 found it overwhelming at times, which is why she had no desire to take Paro outside her home. "Everyone wants you to constantly turn him on to see what he was doing... All that interaction would overwhelm me." (P1) She found it manageable when one person approached her at a time but did not want multiple people approaching. Three participants did take Paro outside the home (even though instructed not to) and enjoyed the additional attention it brought.

P1's interpretation of Paro as animate actually seemed to undercut the relief Paro was meant to provide, as she saw it as a burden. "It's like a dog, too much attention... To keep him alive, I have to interact with him." (P1) She expressed that her own dog was too much responsibility as well. For most participants however, viewing Paro as animate, even though they logically knew it was just a robot, was beneficial. Participants often interacted with Paro together with other people and/or their pets, enhancing the role Paro could play in increasing socialization.

The activities people reported performing with Paro were fairly similar. All participants talked to Paro, engaging in small talk or sharing their deeper thoughts and concerns with it. Even participants who had a social network to turn to did this, as they didn't always feel like 
they could talk to others about their issues, or members of their network where actually the source of their stress. For instance, P10 used Paro to comfort her when she was nervous about her pregnant daughter and her daughter's boyfriend being at her house. Petting Paro (done reflexively or in dealing with stress) was also common. Other types of physical interaction, such as brushing and cuddling it, also occurred but were mentioned to a lesser degree. Participants additionally spent a lot of time interacting with Paro together with other people $(\mathrm{N}=9)$ or their pets if they had them $(\mathrm{N}=4)$, and also slept with the SAR $(\mathrm{N}=4)$. Six participants watched TV with Paro. For some of these participants, this was their primary way of interacting with the SAR.

Participants said the reasons for interacting with Paro were either their perceptions of Paro needing interaction or feeling that they themselves wanted to interact. P9 said, "it's something to do, like babysitting". Her reason for interacting was mainly due to boredom or desiring companionship. On the other hand, P8 would interact often because she felt guilty that Paro wasn't getting enough attention. "[He] looked lonely." In fact, several participants expressed a sense of guilt when they thought they did not engage enough with the robot.

Designing a robot that is lifelike seems to be beneficial in increasing interaction for most older adults. Due to their needs for physical proximity, it is also important that they be able to interact physically with the robot in many ways (e.g., touching, cuddling, holding). This fosters their interpretation of robots as companions, something most older adults with depression are lacking in their lives. Even $\mathrm{P} 1$, who was overwhelmed by Paro being in her home, actually experienced a major decrease in depressive symptoms during its use [4]. It is possible that having to care for something else pushed her to be more active, resulting in increased well-being, although it caused her to view the robot itself somewhat negatively. This call to care-taking was mentioned by therapists as being potentially advantageous for their depressive patients.

Though companionship has been suggested in many studies to be important when designing robots for inhome use for older adults [20,59], our month-long study also revealed that this was not sufficient for many older adults (even depressive older adults, who are ostensibly more sensitive to the need of companionship) to maintain high levels of interaction long-term (see Figure 2). Participants' comments suggested that companionship should be combined with more utilitarian role(s) the robot can also serve. This represents divergence from results usually found from institutional use of Paro, where companionship works well when it is the only functionality present.

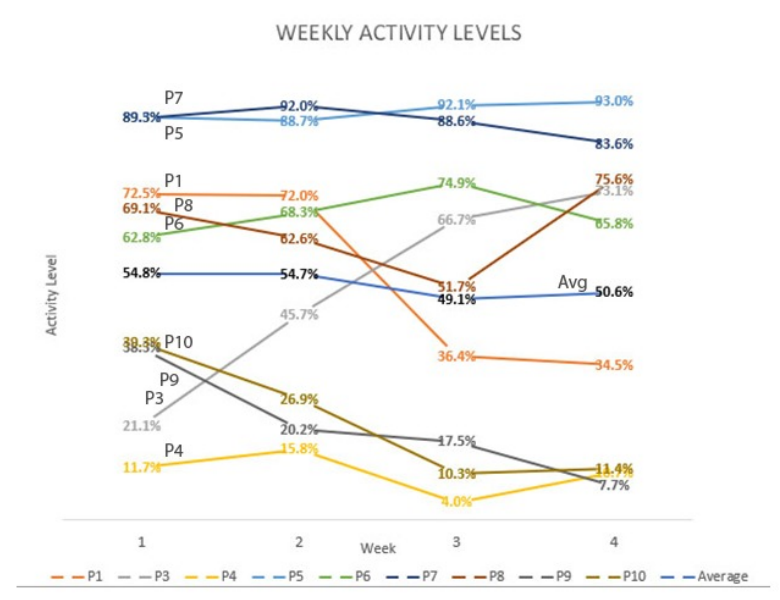

Figure 2: Weekly activity (interaction) percent per participant, as registered by sensors on the robot's collar. The average across all participants is shown in the middle of the graph, in blue.

Possible functions, in line with the needs of depressive older adults specifically, and as expressed in focus groups by older adults and clinical staff and reinforced through our in-home implementation, are elucidated below.

\subsubsection{Call contact(s) when not well}

During the initial workshops, both older adults and clinical care staff mentioned wanting to enable the robot to contact a hospital or designated emergency contact if the owner was not well. Through the in-home study, we found this feature would be well aligned with the daily struggles of participants. Participants' level of depression fluctuated throughout the study, with common slips back into moderate or major depression. While several participants reported that Paro helped them when they experienced symptoms of depression, it was also more difficult for them to voluntarily interact when they slipped into major depression. During these times, it would be good for patients to have an easy lifeline to the outside world. This might be triggered by the patients themselves, or when sensors detect activity that is suggestive of a major depressive episode.

Aside from mental health issues, participants frequently battled physical ailments, including constant and reoccurring issues such as afib, lymphatic system problems, pain, and seizures and temporary sickness from minor flu-like illness to violent illnesses that landed one participant in the emergency room. Allowing participants to easily contact the hospital or a designated contact (possi- 
bly via a button on the robot), or triggering a call based on concerning changes in sensor activity, could help ensure they get the help they need in a timely manner.

\subsubsection{Reminders}

Older adults had a number of therapy and doctor's appointments. They took medication for their depression. Many also took medication for some physical issue. As older adults expressed they would benefit from a physical system providing reminders in the initial focus groups, reminders might be an appropriate supplemental function for a home companion robot to provide to this population. This is something that has been found in previous studies with older adults [60, 61], and seems to be true for depressive older adults as well. Participants kept Paro in the same room with them, so it is possible that a companion SAR could express these reminders verbally (by using a distinct sound or cry) or visually.

\subsubsection{Security system}

As participants were low-income, some lived in more crime prone areas. This was a major reason why they wanted a security system. P6 even discussed someone having recently stolen things from her as part of the in-home interviews during the intervention. Staff feedback from the focus group echoed this concern, as they mentioned wanting the robot to be able to issue alerts in response to security threats. However, due to older adults' concerns about their own privacy, it is not clear that this should be autonomously implemented as part of a robot meant primarily for companionship, as this would most likely entail use of a camera. If older adults are able to use a button or some other mechanism on the robot to alert authorities in response to perceived danger, this may give them a greater sense of perceived security while also respecting their right to privacy. As participants often moved the robot to the same room they were in, this manual alert system is also in line with current use habits. In addition, the robot should potentially be enabled with location tracking, as this solution relates to participants' worry about the robot's cost and its theft.

\subsubsection{Personalization to user}

Versatility and customizability of both the robot and sensors were mentioned repeatedly by both older adults and staff in the initial workshops. Due to participants' diverse circumstances, Paro might best support older adults' home life if it could automatically respond to participants in unique ways. For example, about half of our participants lived with other people, some of whom interacted with Paro regularly. This might make it more important for the robot to be able to recognize individuals and respond to them differently.

As well, some participants (P1, P4, P7, and P10) interacted with Paro less throughout the course of the study (see Figure 2). They said this was because they started finding its interactions more predictable and less interesting. Since Paro seemed to be able to prevent participants from getting sad or help them from spiraling when they experience negative emotions, a home robot meant for this population would need to have more functions or diverse behaviors to keep interaction at moderate levels, or present new behaviors over time to maintain long-term interest and therapeutic benefit. At the conclusion of the focus groups, older adults expressed their willingness to share their data with third party developers for this purpose.

P1 found Paro too demanding, thinking it overwhelming at times, especially when she got "panicky". For her, it may have been better to reduce Paro's attention seeking behaviors, as they caused her anxiety. The ability to lower or turn off Paro's volume would have also been helpful. Even a home robot attempting to spur interaction when it is low may be a function that therapists should have some control over, as this behavior may be helpful for some participants but overwhelming for others.

Personalization may require additional or custom sensors to achieve, with implementation internal or external to the robot. The potential exists to use sensor data to better understand how to optimize the interaction with the user by modulating robotic behavior. This may take the form of increasing the expression of behaviors particularly associated with higher than baseline interaction or by introducing new behaviors when interaction starts to decline.

\subsubsection{Sleep aid and tracking}

Six participants mentioned trouble sleeping at some point during the study. This is also supported by the number of sleep awakenings captured by our sensor data, with approximately 5 awakenings per week for P5 and P8. These participants also had high nighttime circadian activity, with activity levels at $94.1 \%$ and $74.3 \%$, respectively (see Table 3). This is indicative of heavy nighttime interaction with Paro (from the hours of 12am to 6am) for these individ- 
Table 2: The Almere measures attitude toward, and intention to use, a given robot. Higher scores represent more positive attitudes and greater intention to use. Each question is awarded 1-5 points, depending on respondent's answers to a 5-point Likert scale (from "totally disagree" to "totally agree", with a 3 representing a "don't know" option). The maximum possible score in our modified version is 185 .

\begin{tabular}{|l|l|l|l|}
\hline & \multicolumn{2}{|c|}{$\begin{array}{l}\text { Almere score } \\
\text { pre-intervention }\end{array}$} & \multicolumn{1}{l|}{$\begin{array}{l}\text { Almere score } \\
\text { post-intervention }\end{array}$} \\
\hline P1 & 124 & 125 & 1 \\
\hline P2 & 155 & 151 & -4 \\
\hline P3 & 145 & 153 & 8 \\
\hline P4 & 130 & 84 & -46 \\
\hline P5 & 125 & 138 & 13 \\
\hline P6 & 130 & 152 & 22 \\
\hline P7 & 160 & 154 & -6 \\
\hline P8 & 128 & 132 & 4 \\
\hline P9 & 118 & 115 & -3 \\
\hline P10 & 120 & 102 & -18 \\
\hline Mean & 133.5 & 130.6 & -2.9 \\
\hline Median & 129 & 135 & -1 \\
\hline Paired $t$-test & & & $p=.636$ \\
\hline
\end{tabular}

uals. Many participants also reported sleeping more than usual when they were depressed. As well, 4 participants (P2, P5, P6, and P10) slept with Paro at night; it is possible that more participants would have done the same had they not been instructed not to. As Paro was often already in their bedrooms, using it as a sleep aid seems in line with current use habits. As mentioned by therapists, sleep can also go hand in hand with depression, so using it to aid in and track sleep could help in patient care. Potentially, the robot could even help in waking participants up and urging them to get out of bed during particularly low days.

\subsubsection{Identify behavioral patterns}

Participants would often change their routine when they weren't feeling well. As mentioned above, sleep was one area this affected, with participants sleeping longer or not getting out of bed when they were low. This also affected how much they wanted to interact with others and Paro, and led to changes in their regular hygiene habits. "I didn't take care of myself yesterday even. I just had a really hard time getting up and making myself look presentable." (P10) This change in activity level could be a trigger for others to check in on these older adults or for therapists to have a better idea of their patients' emotional states.
Table 3: Sensor captured interaction time and correlation with change in Almere score pre-post in-home deployment of Paro. Activity\% is the total percent of time throughout the day our sensors flagged as interaction, based on the processing described in 3.3.3. Morning\% is from the hours of $6 \mathrm{am}$ to $12 \mathrm{pm}$, afternoon \% from $12 \mathrm{pm}$ to $6 \mathrm{pm}$, evening from $6 \mathrm{pm}$ to $12 \mathrm{am}$, and overnight from $12 \mathrm{am}$ to $6 \mathrm{am}$. The activity\% column was calculated by averaging daily activity readings, while the other columns were calculated by averaging weekly readings.

*P2 was excluded from analysis as she had incomplete sensor data due to sensor malfunction.

\begin{tabular}{|c|c|c|c|c|c|}
\hline & $\begin{array}{l}\text { Activity } \\
\%\end{array}$ & $\begin{array}{l}\text { Morning } \\
\%\end{array}$ & $\begin{array}{l}\text { Afternoon } \\
\%\end{array}$ & $\begin{array}{l}\text { Evening } \\
\%\end{array}$ & $\begin{array}{l}\text { Overnight } \\
\%\end{array}$ \\
\hline P1 & 53.9 & 32.2 & 67.4 & 74.5 & 44.7 \\
\hline P3 & 67.2 & 48.3 & 78.3 & 82.3 & 22.5 \\
\hline P4 & 10.6 & 8.7 & 18.8 & 12.9 & 1.0 \\
\hline P5 & 90.8 & 87.6 & 89.1 & 92.1 & 94.1 \\
\hline P6 & 68.0 & 32.2 & 67.4 & 74.5 & 44.7 \\
\hline P7 & 87.0 & 85.5 & 92.1 & 94.9 & 40.3 \\
\hline P8 & 64.8 & 69.1 & 65.0 & 60.5 & 74.3 \\
\hline P9 & 20.9 & 19.6 & 35.7 & 29.9 & 1.0 \\
\hline P10 & 22.0 & 22.1 & 37.8 & 35.8 & 2.2 \\
\hline $\begin{array}{l}\text { Corr. } \\
\text { Almere } \Delta\end{array}$ & $\begin{array}{l}0.73 \\
(p=.026)\end{array}$ & $\begin{array}{l}0.50 \\
(p=.176)\end{array}$ & $\begin{array}{l}0.73 \\
(p=.026)\end{array}$ & $\begin{array}{l}0.75 \\
(p=.021)\end{array}$ & $\begin{array}{l}0.63 \\
(p=.071)\end{array}$ \\
\hline
\end{tabular}

\subsection{Robot perception survey results}

\subsubsection{Aggregate level}

The Almere measures attitude toward, and intention to use, a given robot (Paro in this case). There were no significant differences pre-post intervention in Almere scores for our participants (see Table 2). The Godspeed questionnaire measures robot perception, looking at five distinct categories: animacy, perceived intelligence, anthropomorphism, likeability, and perceived safety. As with the Almere, there was no significant pre- and postintervention difference in scores on the Godspeed questionnaire when using a paired t-test, $(\mathrm{p}=.776)$. Further analyses, however, suggest robot perception may be affected by pet ownership.

\subsubsection{Pet ownership and robot perception}

Since Paro was often likened to a pet, we analyzed whether there were differences in the association between pet ownership and perception of the robot. While both groups had similar baseline measures, we found that those with pets had decreasing Almere scores, indicating waning positive attitudes toward Paro and intention to use the robot (mean=-8.25). Alternatively, those without pets saw increasing scores (mean=10.33). This was a large effect (Hedges' $\mathrm{g}=1.10, \mathrm{p}=0.099$ ). Those without pets also tended 
to show stable or increasing Godspeed scores (4/4), while those with pets sometimes had markedly reduced scores from their baseline (2/6). This effect of pet ownership on change in Godspeed score was also large (Hedges' $\mathrm{g}=0.86$, $\mathrm{p}=0.191$ ). Our small sample size may likely be a reason these results failed to reach significance. Final Godspeed scores for anthropomorphism, animacy, likeability, and perceived intelligence were higher for those without pets (Figure 3).

Additionally, there was a significant difference between sensor-determined interaction related to pet ownership ( $p=0.014$, Hedges' $g=-1.87$ ), based on sensor data results presented in the subsequent section, indicating that those without pets interacted with Paro more frequently. This result cannot be entirely explained by participants' living status, as there was no difference in perception of Paro, or interaction time with Paro, based on whether participants lived alone, though we note that most participants who had pets also had cohabitants. This points to consistent or possibly increasing interaction and intention to use over time for users without pets.

This may speak to Paro's ability to play a similar role as a pet in individuals' lives. "Pet speak" was very common in our study, comprising over $10 \%$ of coded excerpts in workshops 1 and 3. During the intervention, participants often likened Paro to a pet. One participant mentioned they had gotten a pet since the intervention (due to the intervention), and another was looking. This suggests that Paro is viewed most frequently as a pet, so may influence those without them differently.

\subsection{Sensor captured interaction patterns}

\subsubsection{Interaction time}

Participants interacted with Paro to varying degrees. As can be seen in Table 3, P4, P9, and P10 had the lowest lev-

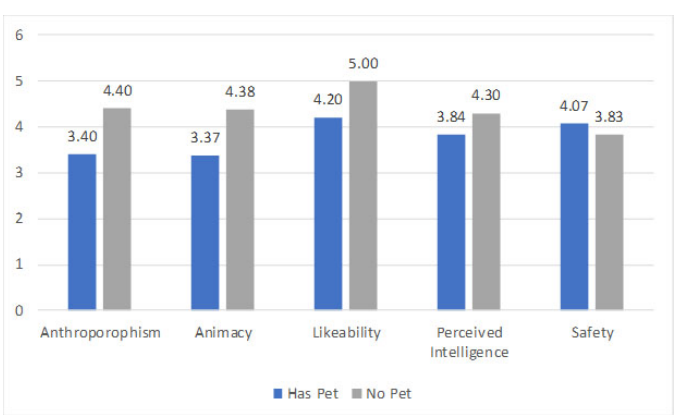

Figure 3: Average Godspeed scores for participants with and without pets. Scores range from 1 to 5 . els of interaction with Paro, according to sensor data. P1, P3, P6 and P8 had moderate levels of interaction. P5 and P7 interacted the most, interacting at consistently high levels throughout the day. As per section 3.3.3, activity was determined based on the presence of multiple simultaneous sensor readings from Paro's collar. Therefore, not all activity necessarily represents direct interaction with the robot. It is the percentage of the time in the given window that activity was registered. Due to sensor malfunction, P2's interaction level could not be determined.

Sensor determined interaction is in line with relative levels of self-reported interaction. There were three common patterns of self-reported interaction frequency. Low interaction represented less than 20 minutes per day or 1 hour per week (P4, P9, and P10). Medium interaction frequency occurred daily or nearly daily, for at least $30 \mathrm{~min}$ utes per day (1-2 hours most common) (P1, P3 and P8). High frequency of interaction occurred daily or nearly daily, for at least four hours a day (P2, P5, P6, and P7). P2, P5, P6, and $\mathrm{P} 10$ also reported occasionally sleeping with Paro, though they had been instructed not to do so for safety reasons. These patterns naturally emerged from the data and were therefore defined during analysis. During week 2, P10's reported interaction decreased from medium to low levels of interaction, and during week 4, P1's interaction exhibited the same decrease. These decreases are supported by sensor readings (see Figure 2).

\subsubsection{Circadian patterns and intention to use}

Sensor data allowed us to determine when participants interacted with Paro during the course of their day. Across all participants, interaction was most common in the afternoon and evening, though the percentage of time participants interacted during all hours varied considerably. Overall interaction was strongly correlated with changes in the Almere scale (Table 3) pre-post in-home intervention (0.73), even though changes in the Almere scale themselves were not significant (Table 2). However, there was an even stronger correlation between certain Circadian patterns of interaction and changes in the Almere scale. In particular, people who interacted with Paro more during the afternoon and evening hours had higher increases in Almere score, representing a greater increase in positive attitudes toward Paro and intention to use the robot (see Table 3). Additionally, we found that individuals with high levels of overnight use tended to be those with lower levels of baseline functioning as per the OQ45 (correlation = $0.58, p=.094$ ). 
In addition to the correlational analysis between Circadian interaction and changes in Almere scores, we also attempted to build a machine learning model to predict changes in Almere scores using various features as described in section 3.3.3. However, we had limited success, due to our small sample size. The RMSE of our predictions was not significantly different from the absolute standard deviation of changes in Almere scores, indicating that our model was performing at nearly chance levels. However, Circadian Rhythm interaction levels were the features identified as most important in this analysis (using the Relief-F algorithm), which supports the results of the correlation analysis.

\section{Discussion}

\subsection{Designing SARs for in-home use}

There are not many long-term in-home SAR studies, likely due to the challenges of conducting them. However, people use technology in the home differently than they do in institutions or communities, where there may be checks as to whether use guidelines are adhered to. In the home, participants are likely to use technology in ways that are not expected, and even in ways that are proscribed against. In our study, this took the form of people sleeping with the robot and taking it outside the home. This suggests that either robots need to be designed to constrain this behavior when use occurs in home environments, or these behaviors should be observed and designed for, such that use in this way is safe, and that this additional use may present opportunities for therapy (e.g., sensors on the robot to collect sleep data, or use of the robot as a sleep aid).

The reasons participants interact with robots may also be different than in institutions. For instance, many participants in our study reported interacting out of guilt, as they were solely responsible for Paro. This is not typical in institutions. This may be partially due to bonding that may occur more strongly than in institutions. For instance, 8 of 10 participants named Paro and often said it was their friend or family member. This likely arises because Paro is theirs alone and they also spend much more time with it than they would in institutions. Some participants spent over 6 hours a day with Paro, excluding sleeping with it.

Additionally, personal robots in the home, as opposed to SARs in institutions, present more opportunities for one-on-one interaction, and therefore might need to have more diverse or complex behaviors, and present multiple functionalities. For many older adults, though companion- ship is highly valued, this function alone does not seem sufficient to spur them to incorporate the technology into their lives long-term. Companion robots need to have multiple functions for extended use in the home, and these abilities need to be situated in individuals' daily concerns. These functions might include the robot serving as a security system or allowing participants to contact the hospital or a designated contact (or automatic notification to therapists or a contact based on sensor data in some contexts). Even personalization needs to be designed in ways specific to the home and to this population, such that the robot spurring interaction automatically when it is low, which may be a trigger for some individuals, should be partially controlled by individual therapists. Identification of, and personalization to, unique behavioral patterns is also harder to do in institutions, where many participants may interact with the robot and each individual interacts less often than in homes. Understanding these differences by implementing emergent technologies in a situated environment is fundamental to ensuring safe and engaging interactions.

This speaks to the importance of placing technology in context to help finalize design. While older adults and clinicians were good at verbalizing what they wanted and needed in the initial workshops, in-home implementation allowed us to see how these requests could be implemented more specifically in relation to participants' everyday activities and experiences. It also revealed to participants new insights about desired robot characteristics, allowing them to give more specific feedback on how their ideas could be implemented. Ultimately, this process can support co-design.

As robots are typically time and cost intensive to produce, our study suggests placing "surrogate" technology in the home, even if it lacks many of the features mentioned as desirable, can help gain insight into which design features are most important and how to realize them. This is common in some design fields (known as technology probes) [62] but has not been implemented widely in social robot design. For instance, placing Paro in the home as a representative of companion robots more broadly, even though it wasn't equipped with all the features older adults deemed highly important, allowed us to see if these were really desirable during usage. While many requested features ultimately were, some were seen as less important (environment detection and home control) or undesired (as in the case of multi-person usage) after in-home implementation. This saves resources before needing to build a prototype more specific to the robot's use. This practice also clarifies how to implement requested features (for example, how to best utilize the robot as a security system), 
as they can be situated in how users would best use them in their daily lives and how they can integrate cohesively alongside other features.

\subsection{Sensor data and robotics}

As mentioned, though the information provided by wearable sensors was valuable, this did not work well for our population. Older adults often forgot to put the wearable on, found it difficult to clasp around their wrist, and were forced to be more active in the sensor collection routine. This makes sensors onboard the robot particularly desirable, as they can collect data passively and potentially use that data to update behaviorally. Sensors placed in the user's external environment that require little maintenance are useful in collecting data where the robot cannot or as a triangulation method (for example sleep and activity data). If this information can be fed to the robot directly, or to clinicians, it can ultimately result in clinically helpful changes in the robot's behavior, as desired by both clinicians and older adults. Of course, this data may also make the interaction more enjoyable for users by allowing the robot to learn and exhibit more complex and novel behavior, possibly causing them to spend more time with the robot and receive full benefits of its use.

\subsection{Limitations}

There are several limitations to our study. We had a low sample size, which limited the power of our statistical tests to detect significant effects. Participants also had additional opportunities for social interaction through regular contact with researchers, and our study did not have a control group to tease out the effect this had on feelings of loneliness and companionship versus the effects of the robot in particular. However, as participants specifically described ways in which they used Paro to alleviate feelings of loneliness and depressed mood, we believe our results are suggestive that pursuing this avenue of research is promising. To provide further support for our design suggestions, these changes would need to be made and the technology placed in users' homes, to determine if this improves positive attitudes and use. Furthermore, all our participants were female, which leaves open the question of whether our findings and design suggestions would generalize to male participants.

\section{Conclusion}

Conducting an initial series of workshops with older adults and clinical care staff provided a good representation of how a companion SAR might be designed to be useful for older adults with depression. However, long-term in-home use of Paro allowed both researchers and participants to see how requested features might be implemented in the specific context of user homes, where requests for functionality changed after use. It also made clearer how initially requested functionality by older adults and clinical care staff fit into the daily lives of older adults with depression. We found that companionship alone as a feature, while crucial, may not be enough to engage many older adults in long-term use. It is best if more practical functionality (e.g. ability to contact the hospital, offer appointment reminders) accompany the social companionship that the robot provides. Additionally, sensors embedded in the robot may present an easy and robust way to collect and relay information that is useful in clinical care to therapists, allow the robot to adapt, and allow individuals to be more active participants in their own health and behavior management. In our study, sensor-determined interaction levels were in line with relative levels of selfreported interaction, and sensor data allowed us to determine precisely when interaction occurred for each individual. Afternoon and evening interaction had the highest correlation with positive changes in attitude toward the robot and intention to use the technology. Additionally, we found that those without pets interacted with Paro significantly more than those with pets, and survey data showed they had more positive attitudes toward the SAR. This suggests that older adults with depression who do not have pets might be a particularly relevant population for further research on in-home use of pet-like SARs.

Acknowledgement: This research was supported by Indiana University's Faculty Research Support Program. We would like to thank all our participants for taking part in the study, and Centerstone Research Institute for their assistance with participant recruitment and use of their facilities for conducting workshops. 


\section{References}

[1] E. Broadbent, R. Stafford, B. MacDonald, Acceptance of healthcare robots for the older population: Review and future directions, International Journal of Social Robotics, 2009, 1(4), 319330

[2] J. Broekens, M. Heerink, H. Rosendal, Assistive social robots in elderly care: a review, Gerontechnology, 2009, 8(2), 94-103

[3] A. Tapus, M. J. Mataric, B. Scassellati, Socially assistive robotics [grand challenges of robotics], IEEE Robotics \& Automation Magazine, 2007, 14(1) 35-42

[4] C. C. Bennett, S. Sabanovic, J. A. Piatt, S. Nagata, L. Eldridge, N. Randall, A robot a day keeps the blues away, In: 2017 IEEE International Conference on Healthcare Informatics (ICHI), 2017, $536-540$

[5] H.-M. Gross et al., Robot companion for domestic health assistance: Implementation, test and case study under everyday conditions in private apartments, In: 2015 IEEE/RSJ International Conference on Intelligent Robots and Systems (IROS), 2015, 5992-5999

[6] Y. Kawaguchi, T. Shibata, K. Wada, The effects of robot therapy in the elderly facilities, Alzheimer's \& Dementia: The Journal of the Alzheimer's Association, 2010, 6(4), S133

[7] E. Torta et al., Evaluation of a small socially-assistive humanoid robot in intelligent homes for the care of the elderly, Journal of Intelligent \& Robotic Systems, 2014 76(1), 57-71

[8] D. Fischinger et al., Hobbit, a care robot supporting independent living at home: First prototype and lessons learned, Robotics and Autonomous Systems, 2016, 75, 60-78

[9] A. Coulourides Kogan, K. Wilber, L. Mosqueda, Moving toward implementation of person-centered care for older adults in community-based medical and social service settings: 'You only get things done when working in concert with clients,' Journal of the American Geriatrics Society, 2016, 64(1), e8-e14

[10] M. Dijkers, Community integration: conceptual issues and measurement approaches in rehabilitation research, Topics in Spinal Cord Injury Rehabilitation, 1998, 4(1), 1-15

[11] S. A. Kolakowsky-Hayner, J. Wright, K. Shem, R. Medel, T. Duong, An effective community-based mentoring program for return to work and school after brain and spinal cord injury, NeuroRehabilitation, 2012, 31(1), 63-73

[12] J. A. Piatt, M. Van Puymbroeck, M. Zahl, J. P. Rosenbluth, M. S. Wells, Examining how the perception of health can impact participation and autonomy among adults with spinal cord injury, Topics in Spinal Cord Injury Rehabilitation, 2016, 22(3), 165-172

[13] P. Ciechanowski et al., Community-integrated home-based depression treatment in older adults: a randomized controlled trial, Jama, 2004, 291(13), 1569-1577

[14] M. C. F. Plati, P. Covre, K. Lukasova, E. C. de Macedo, Depressive symptoms and cognitive performance of the elderly: relationship between institutionalization and activity programs, Revista Brasileira de Psiquiatria, 2006, 28(2), 118-121

[15] H. Robinson, B. MacDonald, N. Kerse, E. Broadbent, The psychosocial effects of a companion robot: a randomized controlled trial, Journal of the American Medical Directors Association, 2013, 14(9) 661-667

[16] A. Kristoffersson, A. M. Loutfi, S. Coradeschi, User-centered evaluation of robotic telepresence for an elderly population, In: 2nd International Workshop on Designing Robotic Artefacts with User-And Experience-Centered Perspectives, 2010

[17] K. Wada, T. Shibata, T. Saito, K. Sakamoto, K. Tanie, Psychological and social effects of one year robot assisted activity on elderly people at a health service facility for the aged, In: Proceedings of the 2005 IEEE International Conference on Robotics and Automation (ICRA), 2005, 2785-2790

[18] L. D. Riek, Healthcare robotics, Communications of the ACM, 2017, 60(11), 68-78

[19] H. R. Lee et al., Steps toward participatory design of social robots: mutual learning with older adults with depression, In: 2017 12th ACM/IEEE International Conference on Human-Robot Interaction (HRI), 2017, 244-253

[20] S. Šabanović, W.-L. Chang, C. C. Bennett, J. A. Piatt, D. Hakken, A robot of my own: participatory design of socially assistive robots for independently living older adults diagnosed with depression, In: International Conference on Human Aspects of IT for the Aged Population, 2015, 104-114

[21] N. Randall, S. Šabanović, W. Chang, Engaging older adults with depression as co-designers of assistive in-home robots, In: Proceedings of the 12th EAI International Conference on Pervasive Computing Technologies for Healthcare, 2018, 304-309

[22] M. R. DiMatteo, H. S. Lepper, T. W. Croghan, Depression is a risk factor for noncompliance with medical treatment: metaanalysis of the effects of anxiety and depression on patient adherence, Archives of Internal Medicine, 2000, 160(14), 21012107

[23] S. J. Bartels, J. A. Naslund, The underside of the silver tsunami - older adults and mental health care, New England Journal of Medicine, 2013, 368(6), 493-496

[24] A. T. Beekman, E. de Beurs, A. J. van Balkom, D. J. Deeg, R. van Dyck, W. van Tilburg, Anxiety and depression in later life: cooccurrence and communality of risk factors, American Journal of Psychiatry, 2000, 157(1), 89-95

[25] K. B. Adams, S. Sanders, E. Auth, Loneliness and depression in independent living retirement communities: risk and resilience factors, Aging \& Mental Health, 2004, 8(6), 475-485

[26] K. M. Clark Cline, Psychological effects of dog ownership: Role strain, role enhancement, and depression, The Journal of Social Psychology, 2010, 150(2), 117-131

[27] T. Majić, H. Gutzmann, A. Heinz, U. E. Lang, M. A. Rapp, Animalassisted therapy and agitation and depression in nursing home residents with dementia: a matched case-control trial, The American Journal of Geriatric Psychiatry, 2013, 21(11), 10521059

[28] S. M. Rabbitt, A. E. Kazdin, B. Scassellati, Integrating socially assistive robotics into mental healthcare interventions: Applications and recommendations for expanded use, Clinical Psychology Review, 2015, 35, 35-46

[29] M. R. Banks, L. M. Willoughby, W. A. Banks, Animal-assisted therapy and loneliness in nursing homes: use of robotic versus living dogs, Journal of the American Medical Directors Association, 2008, 9(3), 173-177

[30] S. C. Kramer, E. Friedmann, P. L. Bernstein, Comparison of the effect of human interaction, animal-assisted therapy, and AIBOassisted therapy on long-term care residents with dementia, Anthrozoös, 2009, 22(1), 43-57

[31] G. Colombo, M. D. Buono, K. Smania, R. Raviola, D. De Leo, Pet therapy and institutionalized elderly: a study on 144 cognitively unimpaired subjects, Archives of Gerontology and Geriatrics, 2006, 42(2), 207-216 
[32] M. C. Le Roux, R. Kemp, Effect of a companion dog on depression and anxiety levels of elderly residents in a long-term care facility, Psychogeriatrics, 2009, 9(1), 23-26

[33] S. D. Hollon, R. B. Jarrett, A. A. Nierenberg, M. E. Thase, M. Trivedi, A. J. Rush, Psychotherapy and medication in the treatment of adult and geriatric depression: which monotherapy or combined treatment?, The Journal of Clinical Psychiatry, 2005, 66(4), 455-468

[34] S. Pampallona, P. Bollini, G. Tibaldi, B. Kupelnick, C. Munizza, Combined pharmacotherapy and psychological treatment for depression: a systematic review, Archives of General Psychiatry, 2004, 61(7), 714-719

[35] H.-M. Gross et al., Progress in developing a socially assistive mobile home robot companion for the elderly with mild cognitive impairment, In: 2011, IEEE/RSJ International Conference on Intelligent Robots and Systems (IROS), 2011, 2430-2437

[36] K. Wada, T. Shibata, T. Asada, T. Musha, Robot therapy for prevention of dementia at home, Journal of Robotics and Mechatronics, 2007, 19(6), 691-697

[37] A. Liang et al., A pilot randomized trial of a companion robot for people with dementia living in the community, Journal of the American Medical Directors Association, 2017, 18(10), 871-878

[38] S. Coradeschi et al., Giraffplus: Combining social interaction and long term monitoring for promoting independent living, In: 2013 6th International Conference on Human System Interactions (HSI), 2013, 578-585

[39] B. Graf, M. Hans, R. D. Schraft, Care-O-bot II-Development of a next generation robotic home assistant, Autonomous Robots, 2004, 16(2), 193-205

[40] A. M. Adami, M. Pavel, T. L. Hayes, C. M. Singer, Detection of movement in bed using unobtrusive load cell sensors, IEEE Transactions on Information Technology in Biomedicine, 2010, 14(2), 481-490

[41] H. Aghajan, J. C. Augusto, C. Wu, P. McCullagh, J.-A. Walkden, Distributed vision-based accident management for assisted living, In: International Conference on Smart Homes and Health Telematics, 2007, 196-205

[42] M. R. Song, Y.-S. Lee, J.-D. Baek, M. Miller, Physical activity status in adults with depression in the National Health and $\mathrm{Nu}$ trition Examination Survey, 2005-2006, Public Health Nursing, 2012, 29(3), 208-217

[43] C. Galambos, M. Skubic, S. Wang, M. Rantz, Management of dementia and depression utilizing in-home passive sensor data, Gerontechnology: International Journal on the Fundamental Aspects of Technology to Serve the Ageing Society, 2013, 11(3), 457-468

[44] K. Wild, L. Boise, J. Lundell, A. Foucek, Unobtrusive in-home monitoring of cognitive and physical health: Reactions and perceptions of older adults, Journal of Applied Gerontology, 2008, 27(2), 181-200

[45] M. E. Pollack et al., Autominder: An intelligent cognitive orthotic system for people with memory impairment, Robotics and $\mathrm{Au}$ tonomous Systems, 2003, 44(3-4), 273-282

[46] K. Caine, S. Šabanovic, M. Carter, The effect of monitoring by cameras and robots on the privacy enhancing behaviors of older adults, In: Proceedings of the Seventh Annual ACM/IEEE International Conference on Human-Robot Interaction (HRI), 2012, 343-350
[47] C. T. Gualtieri, L. G. Johnson, Age-related cognitive decline in patients with mood disorders, Progress in NeuroPsychopharmacology and Biological Psychiatry, 2008, 32(4), 962-967

[48] R. C. Kessler, K. A. McGonagle, M. Swartz, D. G. Blazer, C. B. Nelson, Sex and depression in the National Comorbidity Survey I: Lifetime prevalence, chronicity and recurrence, Journal of Affective Disorders, 1993, 29(2), 85-96

[49] M. M. Weissman, G. L. Klerman, Sex differences and the epidemiology of depression, Archives of General Psychiatry, 1977, 34(1), 98-111

[50] W.-L. Chang, S. Šabanović, Interaction expands function: Social shaping of the therapeutic robot PARO in a nursing home, In: Proceedings of the Tenth Annual ACM/IEEE International Conference on Human-Robot Interaction, 2015, 343-350

[51] C. Bartneck, D. Kulić, E. Croft, S. Zoghbi, Measurement instruments for the anthropomorphism, animacy, likeability, perceived intelligence, and perceived safety of robots, International Journal of Social Robotics, 2009, 1(1), 71-81

[52] M. Heerink, B. Kröse, V. Evers, B. Wielinga, Assessing acceptance of assistive social agent technology by older adults: the Almere model, International Journal of Social Robotics, 2010, 2(4), 361-375

[53] H. R. Lee, Collaborative Design for Intelligent Technologies, PhD Thesis, Indiana University, 2017

[54] J. Guiry, P. Van de Ven, J. Nelson, Multi-sensor fusion for enhanced contextual awareness of everyday activities with ubiquitous devices, Sensors, 2014, 14(3), 5687-5701

[55] K. Lee, M.-P. Kwan, Physical activity classification in free-living conditions using smartphone accelerometer data and exploration of predicted results, Computers, Environment and Urban Systems, 2018, 67, 124-131

[56] I. H. Witten, E. Frank, M. A. Hall, C. J. Pal, Data Mining: Practical Machine Learning Tools and Techniques, Morgan Kaufmann, 2016

[57] C. Bennett, T. Doub, Data mining and electronic health records: Selecting optimal clinical treatments in practice, DMNI, 2010

[58] I. Kononenko, Estimating attributes: analysis and extensions of RELIEF, In: European Conference on Machine Learning, 1994, 171-182

[59] H. Robinson, B. MacDonald, E. Broadbent, The role of healthcare robots for older people at home: A review, International Journal of Social Robotics, 2014, 6(4), 575-591

[60] N. Randall, S. Šabanović, W. Chang, Engaging older adults with depression as co-designers of assistive in-home robots, In: Proceedings of the 12th EAI International Conference on Pervasive Computing Technologies for Healthcare, 2018, 304-309

[61] C.-A. Smarr, A. Prakash, J. M. Beer, T. L. Mitzner, C. C. Kemp, W. A. Rogers, Older adults' preferences for and acceptance of robot assistance for everyday living tasks, In: Proceedings of the Human Factors and Ergonomics Society Annual Meeting, 2012, 56, 153-157

[62] H. Hutchinson et al., Technology probes: inspiring design for and with families, In: Proceedings of the SIGCHI Conference on Human Factors in Computing Systems, 2003, 17-24 